\title{
El desarrollo profesional docente derivado de la aplicación de los Proyectos de Trabajo en un aula infantil. Un estudio de caso
}

\section{Teacher professional development arising from the implementation of the Project Approach in a pre-school class. A case study}

\author{
María del Carmen RUIZ TORRES y Rosario MÉRIDA SERRANO \\ Universidad de Córdoba
}

Recibido: Septiembre 2014

Evaluado: Noviembre 2014

Aceptado: Noviembre 2014

\begin{abstract}
Resumen
Este artículo presenta un estudio etnográfico desarrollado en un aula infantil. Se trata de una investigación cualitativa que focaliza su indagación en la capacidad de los Proyectos de Trabajo para contribuir al desarrollo profesional de la docente. Se analiza si el rol que la docente adopta al trabajar por Proyectos de Trabajo facilita su desarrollo profesional, ya que le ayuda a mejorar las estrategias de atención a la diversidad del alumnado y sus familias. Los resultados obtenidos arrojan los sentimientos de incertidumbre que experimenta la docente y sus dificultades para transitar desde la metodología tradicional hasta la implantación de Proyectos de Trabajo. Se muestra el tránsito desde un rol docente directivo hasta la asunción de un papel de acompañante en el aprendizaje del alumnado. Finalmente, se identifican algunas resistencias en la cultura del centro que ralentizan la implantación de este método tanto en el aula, como su transferencia al resto de etapas educativas del centro.
\end{abstract}

Palabras clave: proyectos de trabajo; educación inclusiva; diversidad; educación infantil; desarrollo profesional docente; estudio de caso.

\begin{abstract}
This article presents an ethnographic case-study developed in a preschool classroom. It is a qualitative research that focuses its inquiry into the ability of the Project Approach to contribute to the professional development of the teacher. It examines if the role adopted by the teacher to work with Project Approach facilitates her professional development because it helps her to improve the strategies of attention to the diversity of students and their families. The results show the feelings of uncertainty that the teacher experiences and her difficulties to shift from the traditional methodology to the implementation of the Project Approach. A transit from a management teacher role towards assuming the role of companion in student learning is shown.
\end{abstract}


Finally, we identify some resistance in the school culture that slow down the implementation of this method both in the classroom as its transfer to other educational levels of the center.

Keywords: project approach; inclusive education; diversity; childhood education; teacher professional development; case study.

La siguiente investigación surge de la participación de una de las autoras en la red RIECU ${ }^{1}$ (Red de Infantil, Escuela-Centro de Formación del ProfesoradoUniversidad) y su labor en torno a los Proyectos de Trabajo (PT en adelante) en la etapa de Educación Infantil. De este modo, nos encontramos ante la necesidad de investigar si los PT son una metodología innovadora que tiene en cuenta a todo el alumnado presente en las aulas infantiles y, con ello, la forma en que influye en el desarrollo profesional de la docente que trabaja por PT.

\section{La Diversidad como característica connatural}

Las características que mayoritariamente tiene una sociedad se perciben como rasgos normativizados, es decir, que posee la mayoría y, por consiguiente, definen sus estándares de "normalidad"'. Sin embargo, junto a estos estándares de referencia, cada ser humano tiene unas características irrepetibles que lo singularizan y le confieren su capacidad de ser único en su especie. Por tanto, la diferencia es una característica indisociable de la condición humana, que no debe transformarse en desigualdad (Cardona, 2008). Diferencia e igualdad, pues, son dos caras de una misma realidad. Hemos de respetar las diferencias, pero propiciando una igualdad de oportunidades para que todos las personas puedan desarrollar al máximo sus competencias (Gordon, 1991). Sabemos que la igualdad total de oportunidades es difícil de conseguir, pero debemos trabajar para aproximarnos y tratar de lograr que su presencia sea cada vez mayor y se entienda como algo esencial y saludable en una sociedad (Aguado, 2010; Arnáiz y De Haro, 2004).

Podemos distinguir los tipos de diversidad que definen a la persona, tanto de forma individual como dentro de un grupo. En cuanto a las diferencias que podemos observar en las personas de forma individual, mencionamos: la inteligencia, los estilos cognoscitivos y de aprendizaje, el rendimiento académico y la personalidad. En relación a las diferencias que podemos observar entre grupos de personas, citamos: diversidad cultural, de origen socioeconómico, étnica, de género y lingüística. Centrándonos en Puigdellívol (1998, p. 14), podemos definir la diversidad como "una característica presente en todos los componentes de la realidad escolar y afecta al alumnado, profesorado y al propio centro como institución".

\footnotetext{
${ }^{1}$ Este trabajo se desarrolla en el aula de la maestra Ana López, al amparo de un proyecto interinstitucional aprobado por la Delegación Provincial de Educación de Córdoba y el Vicerrectorado de Estudios de Postgrado y Formación Continua de la Universidad de Córdoba, 2012/13.
} 


\section{La Educación Inclusiva en la escuela actual}

La educación y, por ende, la atención a la diversidad en la escuela han recorrido un largo camino de transformación hasta la actualidad, en el que se ha producido multitud de cambios para modificar las actitudes de la sociedad y de los profesionales de la educación, con el fin de conseguir una escuela de calidad para todos (Parrilla, 2002; Torres, 2010). Así, a lo largo de las décadas, la educación ha transitado desde una exclusión total, de todo aquello considerado "diferente", hasta la inclusión, el momento actual en el que nos encontramos.

Cuando hablamos de inclusión a menudo se tiende a pensar en hacer que niños y niñas con discapacidad estén incluidos en sistemas educativos tradicionales y así volvemos a lo que se denomina normalidad-educativa (Sapon-Shevin, 2013). Sin embargo, es necesario explicitar que esta definición es incompleta. Por un lado, los niños y las niñas muestran diferencias en multitud de aspectos, no solo en cuanto a discapacidades. Es decir, podemos encontrar diversidad en cuanto a etnia, sexo, intereses, motivaciones, origen socioeconómico, etc. La inclusión de esta diversidad existente en las aulas infantiles requiere crear una escuela donde se tenga en cuenta las demandas del alumnado, cambiando el currículum y destruyendo las barreras que puedan estar dificultando su adecuada formación. Supone, entre otros, utilizar estrategias como el trabajo colaborativo, potenciar la responsabilidad compartida de la escuela con la familia, formas a docentes comprometidos que sigan mejorando su formación durante su vida laboral, etc. La inclusión, por tanto, requiere que el docente sea un agente activo del proceso de enseñanza-aprendizaje, capaz de sacar a flote las capacidades del alumnado a través de una actitud motivadora, estimulante y de curiosidad permanente ante el conocimiento. Esta nueva función del docente tiene como finalidad capacitar a los aprendices para tomar sus propias decisiones, tanto para su aprendizaje como para las demandas de la sociedad (Cardona, 2008; Susinos y Rodríguez-Hoyos, 2011). Además, hace referencia a la igualdad como la oportunidad de que todos alcancen el máximo nivel de desarrollo de sus competencias, utilizando diferentes estrategias e itinerarios, según las necesidades de cada persona (Cole, 2008).

\section{Los Proyectos de Trabajo}

Los PT tienen como máximo precursor a William H. Kilpatrick a través de su teoría de la Metodología de Proyectos. Sin embargo, los PT, como tal, surgen en la década de los años 80 ante la necesidad de dar al currículum una organización distinta de la que había tenido hasta el momento. Urgía incluir en él contenidos que se asemejaran a la realidad de la escuela y que fueran de interés para el alumnado, de forma que, con su aprendizaje pudieran resolver los problemas reales de su vida. Es decir, algo que le hiciera ver que lo que se aprende en la escuela tiene una gran utilidad para la vida (Hernández, 2000). Además, los PT están fundamentados a partir de dos perspectivas, principalmente. Una de ellas es la teoría socioconstructivista del aprendizaje y la otra, el enfoque globalizador. 
El concepto que subyace a los PT es bastante complejo y no goza de una única definición. Aquí, para clarificar su conceptualización hemos tomado la definición que Mérida, Barranco, Criado, Fernández, López y Pérez (2011, p. 66) establecen:

"Una propuesta de aprendizaje de carácter global, que parte de los intereses del alumnado y se apoya en sus hipótesis de trabajo, articulándose de forma flexible en torno a una temática determinada y permitiendo atender a la diversidad de los aprendices, al desarrollarse en un contexto de interacciones, indagación y actividad permanente".

\section{Características generales de los PT}

Los PT poseen una serie de características que los convierte en una metodología innovadora. Así, los PT demandan que la organización del currículum sea flexible, transdisciplinar y globalizado. La flexibilidad curricular se refiere a la capacidad del currículum de adaptarse a todas las situaciones, donde el tiempo y el espacio no sea algo rígido ni esté prefijado de antemano dando lugar así, a una escuela eficaz para todos (Sales, 2004). Por otro lado, con un currículum transdisciplinar, se pretende el desarrollo de distintas temáticas elegidas por el alumnado, resultado de un diálogo de la realidad acerca de los cambios que se suceden en ella. En el modelo curricular que defienden los PT no se distinguen las asignaturas, sino que todos los conocimientos están relacionados de forma global (Mérida et al., 2011). Mediante los PT se pueden abordar los contenidos de las áreas del currículum de Educación Infantil y tener en cuenta los intereses y motivaciones del alumnado, favoreciendo, de este modo, el aprendizaje (Díez, 2002; Domínguez, 2003; Hernández, 2002; Mérida et al., 2011). También, Los proyectos se desarrollan a partir de ideas y problemas basados en la realidad por lo que suponen esfuerzos para resolver o investigar los dilemas del mundo real (Holm, 2011). Es decir, los PT pretenden aportar funcionalidad a lo que se aprende, haciendo que los contenidos que aprende el alumnado en la escuela le sean de utilidad para su vida, permitiéndole que se desenvuelva de forma cada vez más autónoma, crítica y participativa en el entorno que le rodea (Díez, 2002;Hernández, 2002).

\section{Principales características del docente que enseña a través de los PT}

Los PT suponen un reto para el docente, pues este debe cambiar su actitud. Es necesario que se produzca un replanteamiento de sus funciones. Este debe presentar una mente abierta, pues debe aprender a dar rienda suelta al alumnado ante las situaciones de enseñanza-aprendizaje, debe aprender a callar y a no anticipar las respuestas, adoptando una actitud de escucha activa. También, debe tener una actitud de curiosidad, indecisa, mostrando, al igual que el alumnado, su ignorancia acerca del tema que se trata, etc. Además, el docente debe atender a la motivación que se genera, los significados que se exponen $\mathrm{y}$, todos los sentimientos $\mathrm{y}$ emociones que surjan en el aula en relación al tema que se desarrolla. De esta forma, la actitud que mantenga el docente en el aula servirá de modelo al alumnado 
y le servirá como referencia para contagiarse de una actitud de indagación y cuestionamiento permanente de la realidad (Díez, 2002; Domínguez, 2003; Hernández, 2002). El docente debe evitar la perfección instantánea en el aprendizaje del alumnado y que, en su lugar, desarrolle habilidades a través de la investigación (Clemens, 1999). Del mismo modo, Blumenfeld, Soloway, Marx, Krajcik, Gudzial, y Palinscar (1991) describen diversos casos de aulas donde los PTno producen los resultados a la altura de las expectativas, debido a que las investigaciones llevadas a cabo por el alumnado se dirigen en direcciones improductivas. Por eso, es imprescindible que el docente se convierta en orientador o guía del proceso de aprendizaje del alumnado.

Los PT no solo favorecen la participación del alumnado y del docente sino que consideran de vital importancia la implicación de todos los miembros de la comunidad educativa, especialmente la colaboración de las familias. Sin ellas, difícilmente se podrían desarrollar los PT de forma eficaz, debido a la gran ayuda que prestan para obtener el material necesario para el proyecto, la colaboración en la búsqueda de información, etc. También, las familias colaboran en la resolución de dudas, proponiendo descubrimientos y reflexionando durante el proceso de investigación que se desarrolla (Domínguez, 2003; Mérida et al., 2011).

\section{La metodología de Proyectos de Trabajo en Educación Infantil}

Los niños y niñas de Educación infantil están en interacción permanente con su contexto físico y social. Dependiendo de la planificación y relevancia de las situaciones educativas que se les propongan se favorecerá la conexión entre sus ideas previas y el nuevo material de aprendizaje, alcanzando de este modo aprendizajes significativos. En Educación Infantil, el alumnado se encuentra en un período crítico de su desarrollo presentando una especial predisposición favorable para el aprendizaje. Nuestra responsabilidad como docentes es ajustar pedagógicamente las propuestas de aprendizaje, situándonos en su Zona de Desarrollo Próximo (Vygotsky, 1979).

De esta manera, es un hecho que los objetivos y los contenidos propuestos en la etapa de Educación Infantil se alcanzan de forma más fácil cuando se estructuran y se abordan a través de temáticas que surgen del interés de los pequeños. Muchas veces, las editoriales presentan en sus libros temas que no tienen nada que ver con el día a día en el aula, por lo que se produce una absoluta desconexión entre el entorno del alumnado y los contenidos que deben aprender (Curtis y Carter, 2007). Aunque es verdad que, casi siempre, el docente de infantil es capaz de entusiasmar a su alumnado con los temas que introduce, el protagonismo de estos se consigue de forma más intensa, partiendo de lo que ellos y ellas eligen y no de lo que propone unilateralmente el docente, porque de este modo, tienen una curiosidad y una predisposición favorable para aprender sobre ello (Paniagua y Palacios, 2005). A través de los PT, el alumnado de infantil tiene muchas más oportunidades de explorar fenómenos de gran relevancia para él y ser conscientes de lo que está observando y lo que está aprendiendo (Beneke y Ostrosky, 2000). 


\section{RIECU, Red de Infantil - Escuela - CEP - Universidad: los Proyectos de Trabajo como puente entre la formación docente inicial y continua}

RIECU (Red de Infantil, Escuela, Centro de Formación del Profesorado, Universidad) es una red profesional de aprendizaje que surge en el año 2004 y que se organiza a través de la colaboración de tres instituciones encargadas de la formación docente: la escuela, el Centro de Formación del Profesorado y la Universidad. Por ello, los miembros que dirigen RIECU son profesionales de la educación que se encuentran estrechamente vinculadas con la etapa de Educación Infantil y los PT.

El principal propósito de RIECU es reducir la brecha que, actualmente, existe entre la formación docente inicial y continua, así como mejorar la débil relación de la teoría y la práctica educativa. La labor que desempeña RIECU se corresponde con las competencias profesionales que pretende desarrollar la formación inicial del profesorado y que se exponen en el DECRETO 93/2013, de 27 de agosto, por el que se regula la formación inicial y permanente en la Comunicad Autónoma de Andalucía, así como el Sistema Andaluz de Formación Permanente del Profesorado.

El trabajo de investigación que presentamos se enmarca en este contexto, donde RIECU ha facilitado la conexión con el escenario real donde se ha desarrollado el trabajo de campo y se han obtenido los datos empíricos. Igualmente ha supuesto el soporte necesario para conocer y estrechar vínculos profesionales con la docente de Educación Infantil que nos ha permitido estar presente en su aula y compartir las experiencias educativas que allí se han desarrollado durante dos meses. Del mismo modo, hemos tenido la oportunidad de vivenciar, junto a los niños y niñas de cinco años, la ilusión, sorpresa y alegría de un aprendizaje compartido basado en sus intereses plurales.

\section{Diseño del estudio}

\section{Contexto}

El trabajo de campo se ha desarrollado en una localidad situada a 50 kilómetros de Córdoba. Nos encontramos ante el único centro público de Educación Infantil y Primaria de dicha localidad por lo que acoge a un gran número de alumnado gran diversidad sociocultural. El aula en la que tiene lugar esta investigación es el tercer curso del segundo ciclo de la etapa de Educación Infantil.

\section{Participantes}

Las personas participantes han sido: 19 alumnos y alumnas (11 niños y 8 niñas) de 5-6 años, escolarizados desde los tres años y que están aprendiendo a través de la metodología de PT desde entonces, en su inmensa mayoría. La docente cuenta con una experiencia profesional aplicando PT en Infantil de más de 10 años. Finalmente, tres familias del alumnado, que son las que más se han implicado en el 
desarrollo del PT y tienen más disponibilidad para formar parte de esta investigación.

\section{Hipótesis exploratoria}

Focalizamos nuestra mirada en el siguiente interrogante de investigación: ¿El desarrollo profesional que genera la aplicación de la metodología de PT en un aula de Infantil mejora la atención a la diversidad del alumnado y por tanto los procesos de inclusión educativa en esta etapa?

Desagregamos el interrogante inicial en hipótesis exploratorias más concretas:

- ¿Qué capacidades y sentimientos tiene la docente al cambiar la metodología tradicional por los PT?

- ¿La docente se convierte en una verdadera co-investigadora junto al alumnado al trabajar por PT?

- ¿Existen diferencias en cuanto al desarrollo profesional de la docente desde que comienza a trabajar por PT?

- ¿En qué medida la docente apoya la participación familiar?

- ¿Existen resistencias a la implantación de los PT?

\section{Método}

La investigación que presentamos es de tipo cualitativo, y se desarrolla mediante un proceso etnográfico a través de un estudio de caso. De esta forma, la investigadora permanece en el campo durante dos meses, asumiendo un doble rol como investigadora y como observadora participante, manteniendo así, interacciones permanentes con los niños y niñas como sujetos de investigación (Angrosino, 2012).El enfoque etnográfico nos permite adentrarnos en un contexto natural de aula sin alterar las interacciones normales del alumnado mientras participa en diversas situaciones de enseñanza-aprendizaje, al tiempo que escuchamos la propia voz de los informantes (Anderson, 1991).

\section{Técnicas de recogida de información}

Siguiendo a Stake (2007), la recogida de información se realiza a través de diferentes técnicas, destacando la observación sistemática, las entrevistas a la maestra, al alumnado y a las familias, y la documentación pedagógica y del centro como fuentes de triangulación de los datos. 


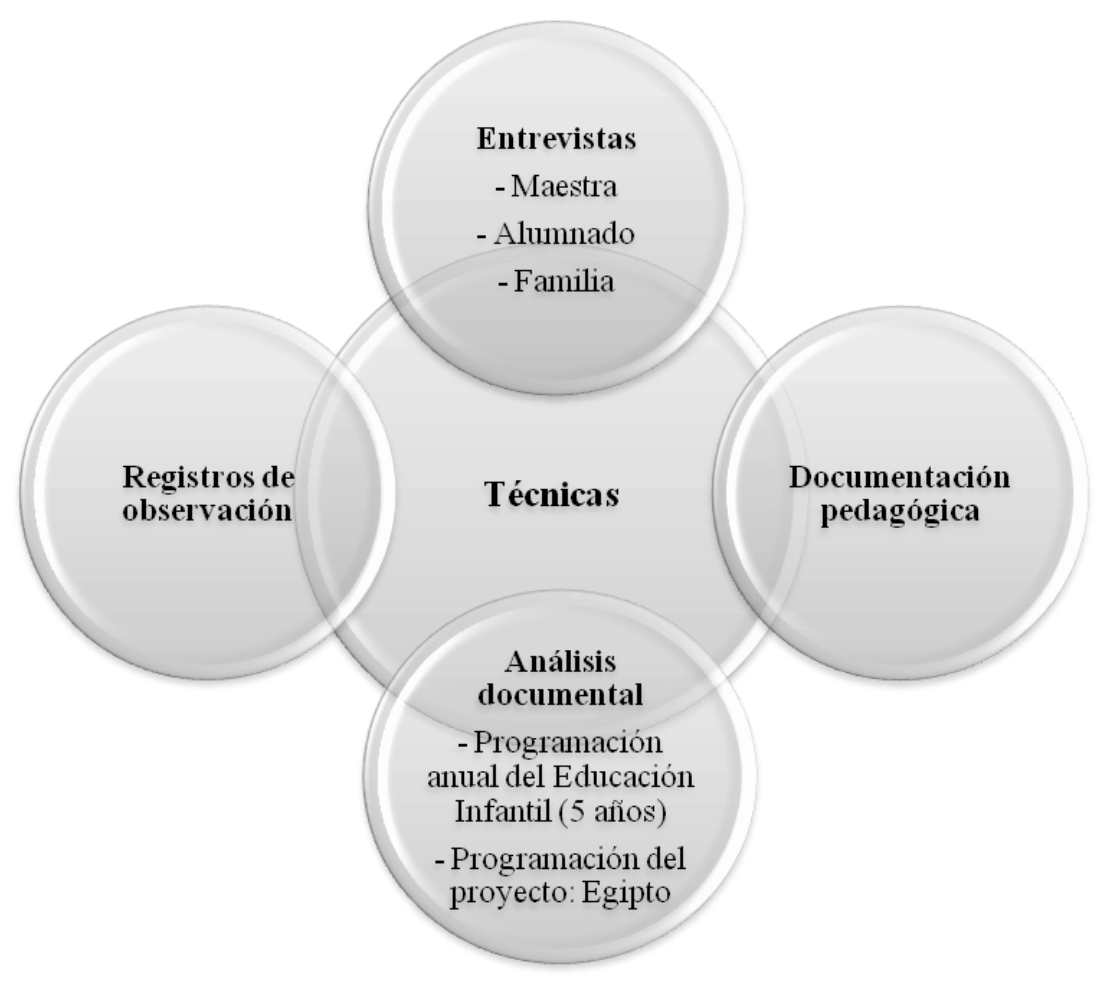

Figura 1. Técnicas utilizadas para la recogida de información.

\section{Técnicas de análisis}

Los datos textuales derivados de las entrevistas, los registros de observación y la documentación del centro, se han analizado mediante análisis del discurso. Por otro lado, los datos visuales obtenidos de la documentación pedagógica (principalmente, fotografías) son analizados a través de un análisis del contenido.

Así, el análisis se desarrolla mediante la construcción de una matriz hermenéutica (T1.) compuesta por las diferentes dimensiones y categorías en las que se agrupan los datos. Su elaboración responde a un proceso mixto (deductivo e inductivo). Partimos de unas dimensiones macro derivadas del marco teórico y que son enriquecidas durante el trabajo de campo y el análisis de los datos con las categorías emergentes que van surgiendo. La posibilidad de trabajo con estos dos tipos de datos permite llevar a cabo una triangulación de los mismos y, así, otorgar la consistencia correspondiente al estudio realizado (Flick, 2004).

El análisis del discurso de los datos textuales se lleva a cabo a través del software informático Atlas.ti 6., tomando como guía la matriz hermenéutica propuesta y elaborando un proceso de codificación concreto. Siguiendo a Gibbs 
(2012), los datos visuales se asignan a la dimensión y categoría correspondiente según el contenido de los mismos.

\begin{tabular}{|c|c|}
\hline DIMENSIONES & CATEGORÍAS \\
\hline 1. Concepto de diversidad & $\begin{array}{l}\text { 1.1. Connatural a la condición humana ("Todos somos } \\
\text { diversos"). }\end{array}$ \\
\hline \multirow{6}{*}{ 2. Tipos de diversidad. } & 2.1. Diversidad de desarrollo y madurez \\
\hline & 2.2. Diversidad de ritmos de aprendizaje \\
\hline & 2.3. Diversidad de intereses y motivaciones \\
\hline & 2.4. Diversidad de origen sociocultural \\
\hline & 2.4.1. Diversidad de origen nacional \\
\hline & 2.4.2. Diversidad de origen socioeconómico \\
\hline \multirow{3}{*}{$\begin{array}{l}\text { 3. Requisitos esenciales de la docente } \\
\text { para trabajar a través de PT }\end{array}$} & 3.1. Apertura mental y flexibilidad \\
\hline & 3.2. Capacidad de observación \\
\hline & 3.3. Creer en el potencial del alumnado \\
\hline \multirow{3}{*}{$\begin{array}{l}\text { 4. Sentimientos de la docente al } \\
\text { iniciarse en los PT }\end{array}$} & 4.1. Miedo \\
\hline & 4.2. Incertidumbre \\
\hline & 4.3. Necesidad de apoyo \\
\hline \multirow{2}{*}{$\begin{array}{l}\text { 5. La docente como co-investigadora } \\
\text { en el PT }\end{array}$} & 5.1. Registro de las ideas erróneas \\
\hline & $\begin{array}{l}\text { 5.2. La experimentación como método de } \\
\text { verificación/rechazo de ideas }\end{array}$ \\
\hline 6. Desarrollo profesional docente & $\begin{array}{l}\text { 6.1. Diferencias entre los inicios en PT de la docente y } \\
\text { la actualidad }\end{array}$ \\
\hline \multirow{2}{*}{$\begin{array}{l}\text { 7, Apoyo a la participación familiar } \\
\text { por parte de la docente }\end{array}$} & 7.1. Aula abierta \\
\hline & 7.2. Actividades realizadas con las familias \\
\hline \multirow{2}{*}{$\begin{array}{l}\text { 8. Resistencias para la implantación } \\
\text { de PT }\end{array}$} & 8.1. Aceptación de los PT por el centro \\
\hline & 8.2. PT vs. Método comercial/tradicional \\
\hline
\end{tabular}

Tabla 1. Matriz hermenéutica

\section{Resultados}

En la primera dimensión, encontramos que la docente es consciente de que el concepto de diversidad es un rasgo connatural de la persona. Ella concibe la diversidad desde su significado más amplio y la identifica con la diferencia y singularidad de cada persona. Además, considera la diversidad como una forma de enriquecimiento para el aula:

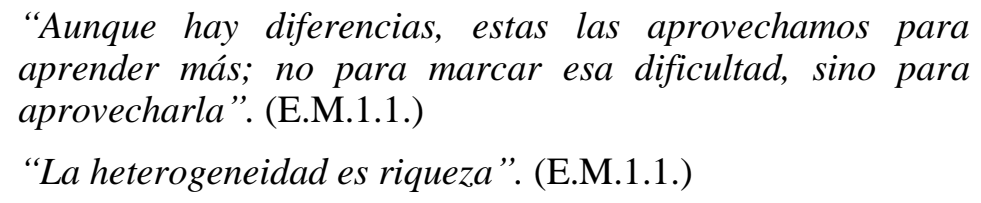

Respecto a la segunda dimensión, podemos observar que la docente tiene conocimiento de los distintos tipos de diversidad que caracterizan a las personas. Esta diversidad hace referencia al desarrollo y madurez, a los distintos ritmos de 
aprendizaje, a las diferentes motivaciones que tienen los niños y niñas y a la diversidad sociocultural de origen nacional y de origen socioeconómico. La docente da muestras de ello de la siguiente forma:

"Nosotros tenemos niños que tienen inteligencias múltiples". (E.M.2.1.)

"No todos son muy expertos, digamos, en lectura o no todos, en creatividad, pero sí hay unos que se les dan muy bien las matemáticas, otros que se les dan muy bien las manualidades....". (E.M.2.1.)

"Cuando hacemos un trabajo hay niños o niñas que pueden llegar más, otros que llegan hasta un cierto límite”. (E.M.2.2.)

"Hay niños que están mucho más entusiasmados que otros dependiendo de los temas, pero bueno eso nos pasa a los adultos también, ¿No?” (E.M.2.3.)

"Cuando vemos lo niños que tenemos que no son españoles, que son de otros países, ellos te dicen, "pues bueno, pues yo en China he visto tal" y a lo mejor estamos investigando de Egipto, pero resulta que sale China y también se aportan cosas". (E.M.2.4.1.)

"Hay familias que pueden acceder a unos recursos y otras que no". (E.M.2.4.2.)

Haciendo referencia a los requisitos esenciales que la docente necesita para trabajar a través de PT, se pueden observar requisitos tales como la apertura mental y la flexibilidad, la capacidad de observación y la creencia en el potencial del alumnado:

"Tienes que tener una mente abierta, porque, claro, tienes que dejar caber en tu clase una serie de elementos que, quien lo ve de fuera, puede decir " $U y$ ! Hay un pequeño caos” o "es permisiva en algunas cosas". (E.M.3.1.)

"Te lleva es que tu programación, que tú la llevas hecha como todas las programaciones, pues tú tengas que coger y decir "bueno pues voy a flexibilizar". (E.M.3.1.)

"La maestra deja que el alumnado se autogestione. Les da autonomía para la asamblea". (D.3.1.)

"Lo que tienes que hacer es ponerte un puntito en la boca y observar muchísimo, dejar a ver qué surge, a ver qué pasa". (E.M.3.2.) 
"Ella está supervisándolo todo desde su mesa o realizando otras acciones aunque, a ojos de ellos, no lo aparente". (D.3.2.)

"A mí me parecía como que se podía sacar más de ellos. Yo creía que había más potencial en un aula, que lo que es llegar y "hazme esta ficha" ". (E.M.3.3.)

"Reconocer que los niños y las niñas tienen un potencial grandísimo que hay que aprovecharlo y que hay que tirar de ahí”. (E.M.3.3.)

Apuntando a la cuarta dimensión, se valoran los sentimientos que surgen en la docente al iniciarse en los PT. Entre ellos destacan el miedo ante la nueva forma de enseñanza-aprendizaje. También, incertidumbre ante la inseguridad que crea un cambio y la duda de si con los PT se cumplirán los objetivos y contenidos marcados para la etapa de Educación Infantil. Por último, señalar la necesidad de apoyo, es decir, la necesidad de tener a alguien a tu lado que sea experto en PT y que te guíe en este nuevo camino:

"Lo primero, miedo. Miedo, porque no sabes si te estás equivocando". (E.M.4.1.)

"La pregunta es : “Me dejaré algún contenido?” “Esto lo estaré haciendo bien?”, “Será una cosa de loca y esto aquí no se me van a enganchar los niños o no van a aprender lo que tienen que aprender?" Eso es lo primero, una incertidumbre que, claro, cuando hay un cambio en tu vida siempre ocurre, siempre hay incertidumbre, pero sobretodo, el cambio". (E.M.4.2.).

"Yendo de la mano de una persona que sabe, pues siempre es más fácil". (E.M.4.3.)

Haciendo mención a la quinta dimensión planteada, los resultados nos muestran que la docente actúa como co-investigadora en el desarrollo de los PT. Ésta se muestra ignorante ante el tema que se trata y acepta los datos erróneos del alumnado en un primer momento para su posterior verificación o rechazo de ideas a través de la experimentación lo que supone una estrategia de aprendizaje en los PT. Así, por un lado, se muestra la aceptación y registro de las ideas erróneas que da el alumnado. Los dicentes aportan todas las ideas que tengan previamente y, aunque no sean correctas, la maestra se lo permite:

\section{"Ellos aprenden que se pueden equivocar". (E.F.5.1.A.) \\ "Saben que si aportan un dato que no es correcto o puede que no lo sea, no pasa nada". (E.M.5.1.)}

Por otro lado, se pone de manifiesto que la experimentación actúa como método de verificación o rechazo de las ideas dadas por el alumnado. Las ideas erróneas del 
aprendiz previamente permitidas, son aceptadas o descartadas y este, a través de la realización de distintas actividades, es consciente de su error o de su acierto:

"Si tú te equivocas, eso forma parte de tú aprendizaje". (E.F.5.2.A.)

"Saben que luego lo vamos a cotejar y lo vamos a comparar y nos vamos a dar cuenta, simplemente, de si era o no era”. (E.M.5.2.)

"De pronto, descubrimos en el libro, que Hatshepsut no era un faraón, como habíamos creído hasta ahora, sino una faraona. Todos los aprendices se sorprenden, pues lo habíamos visto en la lista de faraones que realizó E. y ahora no es así”. (R.5.2.)

En la sexta dimensión, podemos observar el claro desarrollo profesional de la docente, donde ella misma admite sus errores en sus inicios al trabajar por PT cuando echa la vista atrás y la sólida experiencia y conocimientos que presenta en la actualidad:

"El inicio fue un desastre, porque para mí, yo digo "madre mía, ¿cómo hacía yo esto así? iqué dirigido estaba todo!”. Es que, prácticamente, el proyecto lo hacía yo. Y ahora, te das cuenta de que ya, poco a poco, te vas amoldando, vas flexibilizando.... entonces hay una evolución muy grande”. (E.M.6.1.)

"Ahora tengo un formato de programación abierta, flexible, donde cabe que tú aportas, pero no eres el que lleva toda la batuta, digamos, de las cosas. Entonces, ha sido una evolución enorme”. (E.M.6.1.)

En cuanto a la séptima dimensión, en los resultados se puede apreciar que la docente apoya la participación familiar durante todo el proceso de los PT. Por un lado, se puede observar que el aula del alumnado siempre está abierta a las familias para participar en cualquier actividad que se proponga, tanto por parte de ellas como de la docente, aportando información al proyecto en cuestión. Cuando tienen lugar estas situaciones el alumnado muestra respeto por la persona que entra al aula e interés por los datos que aporta para el PT.

"Viene una sabia de Egipto (un familiar) a la clase y todos los niños y niñas se sientan en la alfombra para escuchar lo que tiene que contarnos". (R.7.1.)

“[...] también, estaban las madres preparándolo en el cuarto donde está A. (las cosas que necesita A.) y, entonces lo trajeron para acá a la clase y hacía ta-ta-ta [...]”. (E.A.7.1.B.) 
"Siempre hemos tenido confianza para estar en el aula". (E.F.7.1.B.)

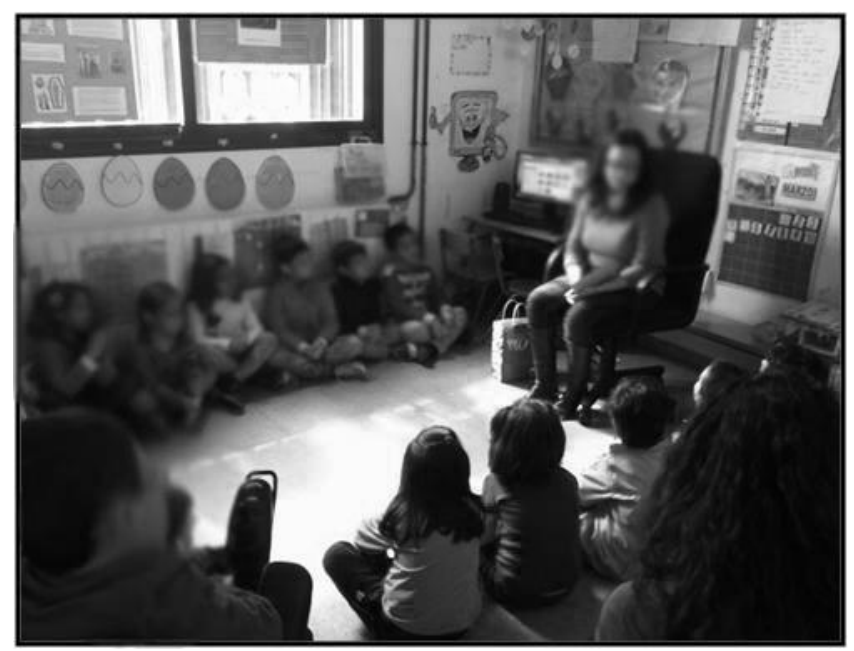

Figura 2. La familia aporta información. En la fotografía se aprecia la entrada de la familia al aula. La sabia de Egipto habla a los niños y niñas sobre su viaje a este país. (V.7.1.)

Por otro lado, se justifica que hay determinadas actividades que son realizadas con la participación de las familias, casi siempre al finalizar el proyecto se prepara algún taller en el que familias e hijos trabajen juntos:

"Con las familias: Talleres; Fiesta final: fiesta de faraones; Visitas de sabios: experta en Egipto”. (P.P.7.2.)

"Elaboración del disfraz de faraones. Por un lado, unas mamás se encargan de hacer el disfraz con bolsas de basura blancas y por otro, otras se distribuyen entre las manchitas para ayudar a los niños y niñas a elaborar los collares para el disfraz". (R.7.2.) 


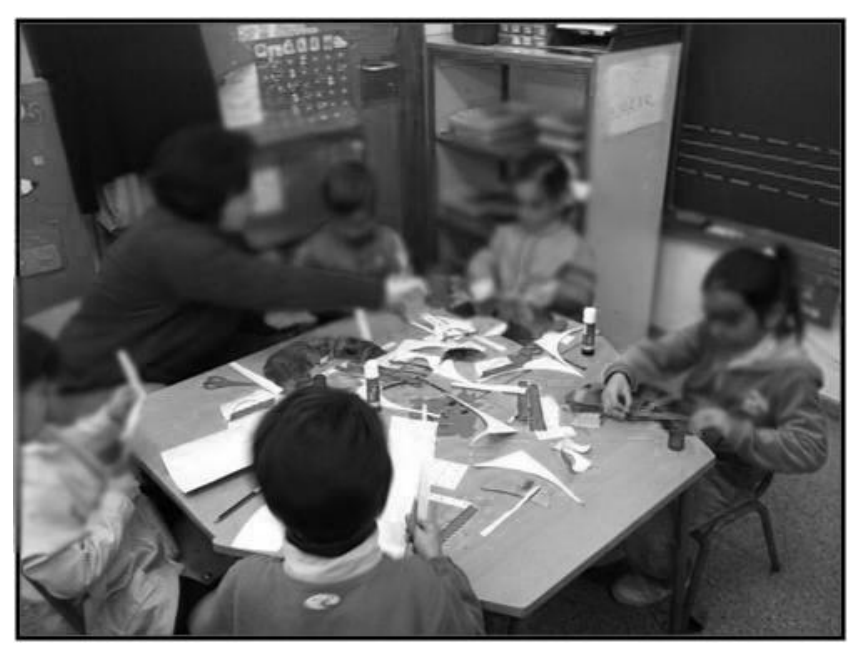

Figura 3. Realización de actividades con la familia. En la fotografía se muestra a una mamá ayudando a los discentes a realizar los collares para el disfraz de faraones y faraonas.

(V.7.2.)

Para finalizar, los resultados muestran una serie de dificultades que surgen, tanto en el centro como en el aula, para favorecer la inclusividad a través de los PT. Una de las dificultades percibidas es la escasa valoración de los PT por parte del centro donde se encuentra la docente que trabaja con esta metodología. Hay centros que dejan libertad de elección de metodología, siempre y cuando haya un consenso entre los niveles educativos que conforman la etapa de Infantil. Sin embargo, en el centro estudiado, pese a que dos de los tres niveles de Infantil trabajan actualmente por PT, no se ha abandonado definitivamente el método comercial, porque supone una forma tradicional de trabajo valorada por las familias y porque genera cierta comodidad en las docentes. Se tratan de culturas de trabajo asentadas y experimentadas durante muchos años que cuesta trabajo modificar:

"La suerte que tenemos es que el equipo directivo nos deja, siempre que haya una coherencia en el nivel. En el momento en que llegase una persona que se negase a hacer proyecto en su aula y quisiera llevar una editorial, tendríamos que llevar editorial. Y lo solaparíamos. Es decir, yo no me voy a negar a hacer lo que se tiene que hacer, pero tampoco me pueden pedir a mí que deje de trabajar en una línea. O sea ni porque van a cambiar ellas, ni porque voy a cambiar yo. Yo pienso que hay que amoldarse un poco." (E.M.8.1.)

"Cuando llegué aquí, ni se conocía lo que era un proyecto ni tenían intención ninguna de cambiar metodologías. Depende de los colegios a los que llegues. Normalmente, ¿Qué 
ocurre? Hay profesionales que están muy seguros de su metodología". (E.M.8.1.)

Otra dificultad que destaca es el tradicional método comercial a partir de la programación hecha por una editorial y la huella tan profunda que ha dejado en los centros y, en concreto, en la etapa de Educación Infantil, que, difícilmente, deja lugar a los PT por la comodidad que produce en el docente. Sin embargo, la docente expone que, a pesar de esa tradición, mantiene su forma de trabajo aunque sea de forma simultánea:

\begin{abstract}
"Cuando llegué ya había un método puesto, un método comercial. Tuve que hacer el método ese año, porque yo no puedo llegar y decir "esto no lo quiero", pero yo iba haciendo proyecto de forma paralela. La gente que iba viendo un poquito, iba diciendo "oye pues me gusta esta forma de trabajo”, “Cómo lo haces?”, te van preguntando, hay otros que, directamente, lo rechazan, porque piensan que es una pérdida de tiempo y para ellos la forma de aprender o de enseñar es ficha, ficha, ficha". (E.M.8.2.)

"Es complicado decir a una persona que, a lo mejor, llevar 15 ó 20 años trabajando de una manera, pues ahora cambia. $Y$ además, te va a decir "pero bueno, si siempre me ha venido bien trabajar así, ¿Por qué tengo que cambiar?”“. (E.M.8.2.)
\end{abstract}

\title{
Discusión y conclusiones
}

Esta investigación pone de manifiesto que el desarrollo profesional que tiene lugar en la docente que trabaja por PT hace posible la inclusión de todo el alumnado y con ello la atención a la diversidad. A continuación, vamos a ir analizando detalladamente las respuestas encontradas acerca de los interrogantes formulados al inicio de esta investigación.

El primer interrogante planteado se refería a la diversidad planteada como algo connatural a la condición humana. La diversidad debe ser entendida como la existencia de características diferentes entre las personas como lo verdaderamente normal y enriquecedor, y no como lo anormal (López Melero, 2004; Muntaner, 2000). Del mismo modo, en los resultados obtenidos, hemos podido apreciar que la docente que trabaja por proyectos considera la diversidad como una condición de la persona $\mathrm{y}$, en sus afirmaciones, expone que aprovecha la riqueza de la heterogeneidad de su aula para desarrollar el proceso de enseñanza-aprendizaje, en consonancia con las aportaciones de Donegan, Hong, Trepanier-Street y Finkelstein (2005).

En respuesta al segundo interrogante, podemos afirmar que la docente es capaz de distinguir entre varios tipos de diversidad en su aula. Esto es apoyado por López Melero (2004) cuando dice que la presencia y reafirmación de las diferencias y la 
anulación de las desigualdades que estas crean en la sociedad suponen el pilar principal para la mejora de la convivencia entre las personas y el avance de las mismas. Esto supone la aceptación de la diversidad en la sociedad como la mejor estrategia para vivir unos con otros desde el respeto mutuo. Al mismo tiempo, Beneke y Ostrosky (2009) afirman que la metodología de PT es una manera eficaz de enseñar al alumnado diverso.

A través de los resultados hemos podido responder a nuestro interrogante acerca de la actitud de la docente ante su decisión para cambiar la metodología tradicional por los PT. Así, se requieren unos requisitos esenciales por su parte. Por un lado, surge la necesidad de un cambio de actitud en el que se produzca una apertura de mentalidad ante el desarrollo del proceso de enseñanza-aprendizaje permitiendo determinadas situaciones que con otras metodologías no se aceptarían. La docente debe dejar de ser una mera transmisora de información y mostrarse como una investigadora más en el aula (López Melero, 2011). En investigaciones de Yuen (2009) se muestra la preocupación de la docente por ser flexible. Además, en los resultados, la maestra afirma que es indispensable ser una buena observadora de todo lo que sucede en el aula para controlar el trascurso que sigue el proceso de enseñanza-aprendizaje, dar cuenta de la evolución del alumnado y detalles que se puedan pasar por alto. Dicha percepción es congruente con las aportaciones de Jones (1992); Rogers \&Sluss (1996) y Vartuli\&Fyfe (1993).

La actitud de la docente al iniciarse en el trabajo por proyectos experimenta una serie de sentimientos y resistencias al cambio... Luna (2008) afirma que los docentes sienten vértigo al inicio de cada curso al verse sin el clásico libro de texto que les guía. En los resultados, se observa que los sentimientos de la docente al comenzar a trabajar por proyectos fueron de miedo, incertidumbre y la necesidad de apoyo por parte de algún profesional en este tipo de metodología. También, Beneke y Ostrosky, (2009) explican que los docentes aprenden a trabajar con PT con la ayuda de otros docentes que les enseñan como si fueran sus mentores. Es decir, se produce lo que se podría llamar "una tutorización entre docentes". El trabajo colaborativo es una de las estrategias más recomendadas en la introducción de cualquier innovación en el aula, ya que el apoyo interpsicológico, el estímulo profesional y las referencias de buenas prácticas de los compañeros, tal y como indican Chard (1998a y 1998b) y Jacobs (1999) suponen algunos de los acicates necesarios para explorar nuevos modelos educativos.

Clark (2006) nos hace partícipes de la diferente forma de desarrollar los PT que tienen los maestros novatos y la de los maestros más experimentados donde estos últimos conceden a los PT un significado más real y profundo que los primeros. Así, los resultados obtenidos dan respuesta a nuestro interrogante acerca del desarrollo profesional de la docente que trabaja con PT donde la propia maestra es consciente de la evolución experimentada desde sus inicios en la aplicación de la metodología hasta el momento actual. En el presente, asume que desempeña un rol mucho más abierto, flexible y de tutelaje del alumnado, alejándose de conductas de transmisión unilateral de sus conocimientos. Esta evolución en el rol docente nos indica que la metodología de PT contribuye al desarrollo profesional, facilitando el 
tránsito paulatino de un rol transmisor a un rol de facilitador del aprendizaje, de forma similar a las propuestas de diversas investigaciones (Katz\&Chard, 1989, Kostelnik, 1991 y Mitchell \& David, 1992).

La importancia de la participación de la familia en la educación de sus hijos e hijas es otro aspecto esencial para incrementar el desarrollo profesional de la docente (Mir, Fernández, Llompart, Oliver, Soler y Riquelme, 2012). Las familias prestan su colaboración en determinadas actividades de los PT y son ellas quienes manifiestan la facilidad que tienen para formar parte del proceso educativo de sus hijos e hijas, y el sentimiento de integración y corresponsabilidad que ello les genera. En este sentido, la maestra valora en gran medida la colaboración de las familias y les da confianza para poder desarrollar los PT, pues estas conocen cuál es su papel en los mismos y se implican afectiva y efectivamente. Asimismo, el encuentro entre familias y profesorado ofrece la posibilidad de poder aprender unos de otros, multiplicar las oportunidades de aprendizaje, ofrecer unas pautas educativas coherentes entre la familia y la escuela y permitir al niño y la niña crecer en un entorno educativo global ajeno a fisuras y contradicciones innecesarias (López Melero, 2011).

Para terminar, debemos hacer referencia a los obstáculos que afectan a la inclusión de una metodología innovadora en los contextos escolares. Nuestros resultados han revelado que la metodología de PT cuenta con una serie de dificultades para poder aplicarse en la etapa de Educación Infantil que tienen que ver con las resistencias a cambiar el rol docente y a poner en marcha procesos educativos inclusivos, globalizados y activos. Se han identificado algunos requisitos necesarios para acometer con éxito la implantación de esta metodología que gozan de un amplio consenso entre los docentes de la etapa Infantil. Sin embargo, la experiencia indica que, en un número de casos más elevado del deseable, triunfa la rutina y el poder de la costumbre instaurada como procedimiento más eficaz e inalterable, el cual se concreta en el uso indiscriminado y excluyente del método comercial. Así, aunque en la literatura científica existen evidencias de los buenos resultados que los PT producen en el aprendizaje integral del alumnado, así como en el desarrollo profesional docente, aún perviven reticencias en la comunidad educativa para asumirlos como forma de trabajo generalizado (Mérida et al., 2011). Como consecuencia, es necesario trabajar de forma sistemática en la formación inicial de los futuros docentes de la etapa infantil las estrategias educativas innovadoras centradas en la actividad del alumnado, mostrando la metodología de PT a los estudiantes universitarios como una herramienta apropiada para transformar los escenarios educativos en contextos estimulantes de aprendizaje que favorecen el desarrollo integral de la infancia (Luna, 2008; Mitchell, Foulger, Wetzel y Rathkey, 2009). 


\section{Referencias bibliográficas}

AGUADO, T. (2010). Diversidad e igualdad en educación. Madrid: UNED.

ANDERSON, G. L. (1991). La validez de los estudios etnográficos: implicaciones metodológicas. En M. Rueda, G. Delgado et, al. (coord.), El aula universitaria. Aproximaciones metodológicas. México: CISE/UNAM, pp. 35-51.

ANGROSINO, M. (2012). Etnografía y observación participante en Investigación Cualitativa. Madrid: Morata.

ARNÁIZ, P. Y DE HARO, R. (2004). Ciudadanía e interculturalidad: claves para la educación del siglo XXI. Educatio, 22, 19-37.

BENEKE, S. Y OSTROSKY, M. (2000). Teachers'View of the efficacy of Incorporating the Project Approach into Classroom Practice with Diverse Learners. Early Childhood Research \& Practice, 11(1), 1-9.

BLUMENFELD, P. C., SOLOWAY, E., MARX, R.W., KRAJCIK, J. S., GUDZIAL, M. \& PALINSCAR, A. (1991). Motivating project-based learning: Sustaining the doing, supporting the learning. Educational Psychologist, 26(3\&4), 269-398.

CARDONA, M. C. (2008). Diversidad y educación inclusiva. Enfoques metodológicos y estrategias para una enseñanza colaborativa. Madrid: Pearson Educación.

CHARD, S. C. (1998a).The project approach: Making curriculum come alive. New York: Scholastic.

CHARD, S. C. (1998b).The project approach: Managing successful projects. New York: Scholastic.

CLARK, A.M. (2006).Changing Classroom Practice to Include the Project Approach. Early Childhood Research \& Practice, $n^{\circ} 2$ (8), 1-9.

CLEMENS, S.G. (1999). “Editing: Permission to start wrong”. Early Childhood Research and Practice, 1(1).[Extraído el 7 de noviembre de 2014 de http://ecrp.uiuc.edu/v1n1/clemens.html]

COLE, R. (2008). Educating Everybody's Children. Association for Supervision and Curriculum Development (ASCD).

CURTIS, D. y CARTER, M. (2007). Learning together with young children: A curriculum framework for reflective teachers. Order from Harvest Resources, P.O. Box 1086, Mechanicsburg, PA 17055.

DECRETO 93/2013, de 27 de agosto, por el que se regula la formación inicia y permanente en la Comunicad Autónoma de Andalucía, así como el Sistema Andaluz de Formación Permanente del Profesorado. BOJA, n 170, 30 de agosto de 2013. [Extraído el 2 de septiembre de 2013 de http://www.juntadeandalucia.es/educacion/educacion/nav/contenido.jsp?pag=/”C ontenidos/IEFP/FORMACION/20130909_DecretoRegulaFormacionProfesorado] 
DÍEZ, C. (2002). La oreja verde de la escuela. Trabajo por proyectos y vida cotidiana en la escuela infantil.Madrid: De la Torre.

DONEGAN, M., HONG, S. B., TREPANIER-STREET, M. \& FINKELSTEIN, C. (2005). Exploring how project work enhances student teachers' understanding of children with special needs. Journal of Early ChildhoodTeacherEducation, 26(1), 37- 46.

DOMÍNGUEZ, G. (2003). En busca de una escuela posible. Revista Interuniversitaria de Formación del Profesorado, 17(3), 29-47.

FLICK, U. (2004). Introducción a la investigación cualitativa. Madrid: Morata.

GIBBS, G. (2012). El análisis de datos cualitativos en investigación cualitativa. Madrid: Morata.

GORDON, E.W. (1991). Human Diversity and Pluralism. Educational Psychologist, 26(2), 99-108.Hernández, F. (2002).Los Proyectos de Trabajo. Mapas de navegantes en mares de incertidumbre. Cuadernos de Pedagogía, 310, 78-82.

HOLM, M. (2011). Project-Based Instruction: A Review of the Literature on Effectiveness in Prekindergarten through 12th Grade Classrooms. InSight: Rivier Academic Journal, 7 (2) , 1-13.

JACOBS, G. (1999). Modeling developmentally appropriate practices: Putting our theory into practice. Journal of Early Education and Family Review, 7, 8-15.

JONES, E. (Ed.). (1992). Growing teachers: Partnerships in staff development. Washington, DC: National Association for the Education of Young Children.

KATZ, L. G., \& CHARD, S. C.(1989). Engaging children's minds: The project approach. Greenwich, CT: Ablex.

KOSTELNIK, M. J. (Ed.). (1991). Teaching young children using themes. Glenview, IL: Scott, Foresman.

MITCHELL, A., \& DAVID, J. (Eds.). (1992). Explorations with young children: A curriculum guide from the Bank Street College of Education. Mt. Rainier, MD: Gryphon House.

LÓPEZ MELERO, M. (2004). Construyendo una escuela sin exclusiones: una forma de trabajar en el aula con proyectos de investigación. Málaga: Aljibe.

LÓPEZ MELERO, M. (2011). Barreras que impiden la escuela inclusiva. Innovación Educativa, 21, 37-54.

LUNA, F. (2008). El alumnado decide qué se va a trabajar. El trabajo por proyectos en el CEP Arrankudiaga Lhi. Cuadernos de Pedagogía, 384, 20-25.

MÉRIDA, R., BARRANCO, B., CRIADO, E., FERNÁNDEZ, N., LÓPEZ, R. M. Y PÉREZ, I. (2011). Aprender investigando en la escuela y en la universidad. 
Una experiencia de investigación-acción a partir del Trabajo por Proyectos. Investigación en la escuela, 73, 65-76.

MIR, M. L., FERNÁNDEZ, V., LLOMPART, S., OLIVER, M.M., SOLER, M.I. Y RIQUELME, A. (2012). La interacción escuela-familia: algunas claves para repensar la formación del profesorado de Educación Infantil. Revista Electrónica Interuniversitaria de Formación del Profesorado, 15(3), 173- 185.

MITCHELL, S., FOULGER, T.S., WETZEL, K., Y RATHKEY, C. (2009). The Negotiated Project Approach: Project-Based Learning without Leaving the Standards Behind. Early Childhood Education Journal, 36, 339-346.

MUNTANER, J.J. (2000). La igualdad de oportunidades en la escuela de la diversidad. Profesorado, Revista de Currículum y Formación de Profesorado, 4(1), 1-17.

PANIAGUA, G. Y PALACIOS, J. (2005). Educación infantil. Respuesta educativa a la diversidad. Madrid: Alianza.

PARRILLA, M.A. (2002). Acerca del origen y sentido de la educación inclusiva. Revista de Educación, 327, 11-29.

PUIGDELLÍVOL, I. (1998). La educación especial en la escuela integrada: una perspectiva desde la diversidad. Barcelona: Graó.

ROGERS, C. S., \& SLUSS, D. J. (1996).Developmentally appropriate practice in higher education.Journal of Early Childhood Teacher Education, 17(1), 4-14.

SALES, A. (2004). Hacia una escuela inclusiva intercultural: los proyectos de trabajo como propuesta curricular y formativa. XXI, Revista de Educación, 6, 139-153.

SAPON-SHEVIN, M. (2013). La inclusión real: Una perspectiva de justicia social. Revista de Investigación en Educación, n 11 (3), 71-85.

STAKE, R.E. (2007). Investigación con estudio de casos ( $4^{a}$ Ed.). Madrid: Morata.

SUSINOS, T. Y RODRÍGUEZ-HOYOS, C. (2011). La educación inclusiva hoy. Reconocer al otro y crear comunidad a través del diálogo y la participación Revista Interuniversitaria de Formación de Profesorado, 25(1), 15-30.

TORRES, J. A. (2010). Pasado, presente y futuro de la atención a las necesidades educativas especiales: Hacia una educación inclusiva. Revista Perspectiva Educacional, 49(1), 62-89.

VARTULI, S., \& FYFE, B. (1993). Teachers need developmentally appropriate practices too. Young Children Y, 48(4), 36-41.

VYGOTSKY, L.S. (1979). El desarrollo de los procesos psicológicos superiores. Barcelona: Crítica. 
YUEN, L.H.F. (2009). From Foot to Shoes: Kindergartners', Families' and Teachers' Perceptions of the Project Approach. Early Childhood Education Journal, 37, 23-33.

\section{Correspondencia con las autoras}

Rosario MÉRIDA SERRANO

María del Carmen RUIZ TORRES

Rectorado de la Universidad de Córdoba

Avda Medina Azahara, ${ }^{\circ} 5$.

14071. Córdoba

e-mail: ed1meser@uco.es 